\title{
Umbolith: a cause of umbilical discharge and omphalitis
}

\author{
Gabriel Rodrigues ${ }^{1}$
}

Received: 10 February 2015 / Accepted: 3 April 2015 / Published online: 5 May 2015

(C) Springer-Verlag Berlin Heidelberg 2015

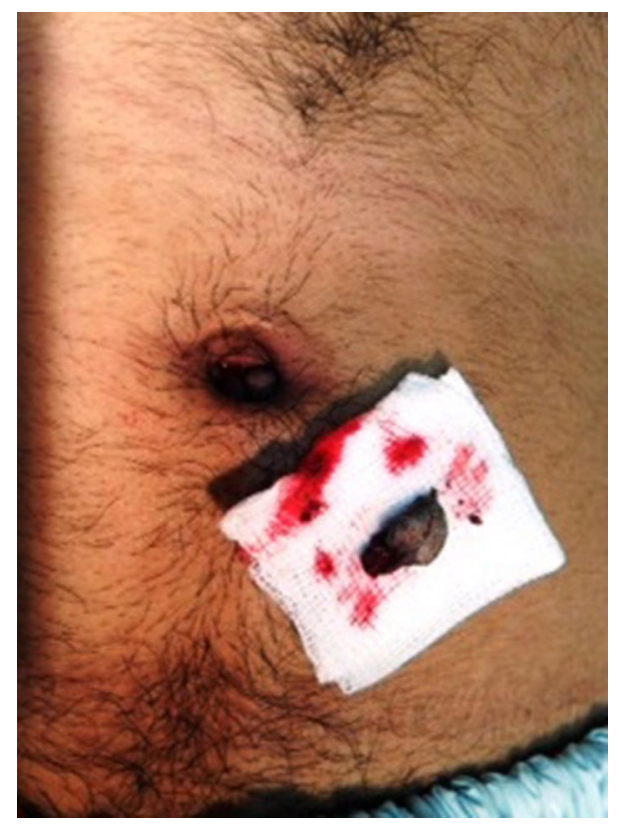

Fig. 1 Picture showing removed umbolith with omphalitis and surrounding cellulitis

A 32-year-old hairy male presented to the out-patient clinic with complaints of pain and foul-smelling bloody discharge from umbilicus of 2 weeks duration. There was no history of any foreign body insertion, systemic symptoms or a similar history in the past. On examination, the umbilicus was deep with periumbilical cellulitis, edema and a foul-smelling bloody discharge. On probing, a 'stony hard

Gabriel Rodrigues

gabyrodricks@gmail.com

1 Specialist General and Laparoscopic Surgeon, Department of General Surgery, NMC Speciality Hospital, Dubai, UAE foreign body' was felt. It was gently separated all around and was scooped out (Fig. 1) and was found to be an umbolith. The underlying abscess was drained and unhealthy tissue debrided. Local antibiotic cream (Fusidic Acid $2 \%$ ) was applied and prescribed. Pus culture grew Staphylococcus aureus which was sensitive to Fusidic Acid. Patient was advised on good personal hygiene and made an uneventful recovery and is well now.

Umbilical discharge in adult is rare, can be quite alarming and could be congenital or acquired, but is usually caused by a foreign material, most commonly hair. Obesity, deep umbilicus and poor hygiene seem to be the predisposing factors. Embryonal anomalies, such as patent urachus/vitelline duct, urachal cyst or sinus, vitelline cyst or sinus may present as umbilical discharge [1]. However, the most common causes of umbilical discharge in adults are acquired conditions: hair tufts with infection, pilonidal sinus and foreign bodies [2]. Rare causes include endometriosis and metastatic carcinoma [3]. Most of the patients are young, hairy males with deep umbilicus with poor personal hygiene, as our patient. Removal of the foreign body with local antibiotic therapy should be done with an advice to proper personal hygiene.

Conflict of interest None.

\section{References}

1. de Silva WD, Samarasinghe MC, Dias MN, Perera CS. Ectopic gastric and pancreatic tissue: a rare cause of umbilical discharge. Ann Trop Paediatr. 2010;30(1):73-5.

2. Sarma DP, Teruya B. 'Lint ball' omphalitis, a rare cause of umbilical discharge in an adult woman: a case report. Cases J. 2009;27(2):7785.

3. Molderez CM, Wouters KB, Bergmans GB, Michiels GK. Umbilical discharge: a review of 22 cases. Acta Chir Belg. 1995;95(3):166-9. 